\title{
0117 PROVIDING PREHOSPITAL TRAUMA CARE FOR ROAD TRAFFIC INJURY VICTIMS IN IRAN: A QUALITATIVE STUDY OF BARRIERS AND FACILITATORS
}

HHBidgoli*,MHasselberg,HKhankeh,DKhorasani-Zavareh,EJohansson Correspondence: Department of Public Health Sciences, Division of Global Health (IHCAR), Karolinska Institute, 17177 Stockholm, Sweden

10.1136/ip.2010.029215.117

Background Road traffic crashes are a major cause of death and injury, especially in low and middle-income countries (LMICs). Improvements in prehospital trauma care can help minimise mortality and morbidity from road traffic injuries (RTIs) worldwide, particularly in LMICs with a high rate of RTIs such as Iran. The aim of current study was to explore barriers to and potential facilitators for providing effective prehospital trauma care for RTI victims in Iran based on the experience and perception of prehospital trauma care professionals.

Methods This study conducted using a grounded theory approach. Participants of the study were 15 prehospital trauma care professionals. The data collected via indepth interviews and were analysed using the constant comparative method. Results Seven categories emerged through data analysis: (1) administration and organisation, (2) staff qualifications and competences, (3) availability and distribution of resources, (4) communication and transportation, (5) involved organisations, (6) laypeople and (7) infrastructures. The lack of interaction and common understanding was identified as the core category. Moreover, a conceptual model was developed based on the categories.

Conclusions According to the findings, improving the interaction within the current prehospital trauma care system and building a common understanding of the role of the emergency medical services were identified as the key issues for developing an effective prehospital trauma care system in the country. 